\title{
Lumbar nerve root injections: a prospective cohort outcomes study comparing age- and gender-matched patients who returned an outcomes-based postal questionnaire with patients who did not return the postal questionnaire
}

Lechmann, Marco ; Peterson, Cynthia K ; Pfirrmann, Christian W A ; Hodler, Jürg

\begin{abstract}
OBJECTIVE: The purpose of this study is to investigate if relying on postal questionnaires returned by patients provides an accurate representation of reported outcomes from patients receiving imaging-guided lumbar nerve root injections (NRIs). MATERIALS AND METHODS: Patients who received imaging-guided transforaminal lumbar NRIs were given short questionnaires inquiring about pain level [numerical rating scale (NRS)] and overall improvement [Patient's Global Impression of Change (PGIC)]. Those who did not return the questionnaires (non-responders) were telephoned and asked about pain level and overall change in condition. Age and gender matching of responders and non-responders resulted in 97 patients in each group. The proportion of patients reporting clinically relevant "improvement" or "worsening" in each group was calculated and the Chi-square test was used to detect differences. NRS and PGIC scores for responders and non-responders were compared using Student's t test and the Mann-Whitney U test, respectively. RESULTS: A higher proportion of non-responders reported clinically relevant improvement $(53.6 \%)$ compared to responders $(42.6 \%)$ and responders reported significantly higher levels of worsening of condition $(\mathrm{p}=0.01)$. Both responders and non-responders had significant (p 0.05) improvement on the 20-30-min and 1-month NRS scores compared to their pre-injection baseline scores. Non-responders had significantly higher baseline NRS scores but no significant difference at the 20-30-min and 1-month NRS scores compared to responders. CONCLUSIONS: Patients returning postal questionnaires reported less favorable outcomes compared to those who did not return their questionnaires.
\end{abstract}

DOI: https://doi.org/10.1007/s00256-013-1673-9

Posted at the Zurich Open Repository and Archive, University of Zurich

ZORA URL: https://doi.org/10.5167/uzh-89819

Journal Article

Published Version

Originally published at:

Lechmann, Marco; Peterson, Cynthia K; Pfirrmann, Christian W A; Hodler, Jürg (2013). Lumbar nerve root injections: a prospective cohort outcomes study comparing age- and gender-matched patients who returned an outcomes-based postal questionnaire with patients who did not return the postal questionnaire. Skeletal Radiology, 42(10):1429-1435.

DOI: https://doi.org/10.1007/s00256-013-1673-9 


\title{
Lumbar nerve root injections: a prospective cohort outcomes study comparing age- and gender-matched patients who returned an outcomes-based postal questionnaire with patients who did not return the postal questionnaire
}

\author{
Marco Lechmann • Cynthia K. Peterson • \\ Christian W. A. Pfirrmann • Jürg Hodler
}

Received: 1 February 2013 /Revised: 22 May 2013 / Accepted: 9 June 2013 / Published online: 16 July 2013

(C) ISS 2013

\begin{abstract}
Objective The purpose of this study is to investigate if relying on postal questionnaires returned by patients provides an accurate representation of reported outcomes from patients receiving imaging-guided lumbar nerve root injections (NRIs).

Materials and methods Patients who received imagingguided transforaminal lumbar NRIs were given short questionnaires inquiring about pain level [numerical rating scale (NRS)] and overall improvement [Patient's Global Impression of Change (PGIC)]. Those who did not return the questionnaires (non-responders) were telephoned and asked about pain level and overall change in condition. Age and gender matching of responders and non-responders resulted in 97 patients in each group. The proportion of patients reporting clinically relevant "improvement" or "worsening" in each group was calculated and the Chi-square test was used to detect differences. NRS and PGIC scores for responders and
\end{abstract}

M. Lechmann • C. K. Peterson $(\bowtie) \cdot$ C. W. A. Pfirmann Department of Radiology, Orthopaedic University Hospital of Balgrist, Forchstrasse 340, 8008 Zurich, Switzerland e-mail: cynthia.peterson@balgrist.ch

M. Lechmann

e-mail: marco.lechmann@gmx.ch

C. W. A. Pfirmann

e-mail: christian.pfirrmann@balgrist.ch

C. K. Peterson

Department of Chiropractic, Orthopaedic University Hospital of Balgrist, Forchstrasse 340, 8008 Zurich, Switzerland

J. Hodler

Department of Radiology, University Hospital, Rämistrasse 100,

8091 Zurich, Switzerland

e-mail: juerg.hodler@usz.ch non-responders were compared using Student's $t$ test and the Mann-Whitney $U$ test, respectively.

Results A higher proportion of non-responders reported clinically relevant improvement (53.6\%) compared to responders $(42.6 \%)$ and responders reported significantly higher levels of worsening of condition $(p=0.01)$. Both responders and non-responders had significant $(p \leq 0.05) \mathrm{im}$ provement on the 20-30-min and 1-month NRS scores compared to their pre-injection baseline scores. Non-responders had significantly higher baseline NRS scores but no significant difference at the 20-30-min and 1-month NRS scores compared to responders.

Conclusions Patients returning postal questionnaires reported less favorable outcomes compared to those who did not return their questionnaires.

Keywords Low back pain · Transforaminal · Epidural . Nerve root block $\cdot$ Pain assessment $\cdot$ Outcomes

\section{Introduction}

Lumbar spine pain is common and a burden not only to the individual but also to the economy as it produces costs for use of the health care system, as well as considerable costs through loss of productive time [1]. Different therapeutic approaches have developed as there are many causes of low back pain.

For the treatment of radicular pain due to compression of a nerve root from disc herniation, degenerative stenosis, or failed back surgery syndrome, lumbar nerve root injections (NRIs), also called lumbar transforaminal epidural injections, are a well-established treatment procedure for patients where conservative care has failed and/or when surgery is not indicated [2-4]. If lumbar NRIs are done for therapeutic purposes, a local 
anesthetic is injected together with a corticosteroid as radicular pain is thought to be generated by both mechanical deformation and inflammatory cytokines [5]. For diagnostic lumbar NRIs, done to determine if a particular nerve root is the source of the pain, only local anesthetics are used.

Although widely used in practice, there remains controversy concerning lumbar NRIs. A recent study by Teske et al. [6] concluded that injection of local anesthetics combined with cortisone showed no advantage compared to injection of local anesthetics alone. However, that study only evaluated the effect on patients with chronic low back pain, not those with acute symptomatology, nor was there a comparison with placebo injections. In addition, Peterson and Hodler [4] note that many studies report different outcomes about the effectiveness of transforaminal epidural injections. This might be due to the fact that there is a great variability in the use of drugs and the method in which success was measured and data retrieved. They conclude that there is some uncertainty about how reliable and accurate effectiveness was measured in the studies and show the need of subgroup analysis, identifying those patients who benefit the most from lumbar NRIs.

Due to the controversy concerning lumbar NRIs, further research is needed to clarify the effectiveness of this procedure [4-7]. It is critical to know whether radiologists and clinicians can rely on the information obtained from patients in research studies and how various methods of data collection may influence the results obtained. Accurate knowledge about the validity of questionnaire data returned by patients will provide more relevant information and is essential for future studies $[4,6,8,9]$.

Starting in June 2009, all patients at this specialized orthopedic/rheumatology hospital who received imagingguided therapeutic musculoskeletal injections were requested to participate in prospective outcomes studies to develop a large database by completing and returning a postal questionnaire. It was noted several months into the creation of this database that a large proportion of the patients failed to return this questionnaire. As lumbar nerve root injections are the most common therapeutic injection procedure performed at this hospital, it was desired to know if those outcomes-based questionnaires returned were a true reflection of the overall patient outcomes from this procedure. Therefore, the purpose of this study is to compare the outcomes from patients who received imaging-guided lumbar transforaminal NRIs who returned an outcomes-based postal questionnaire with patients who did not return the questionnaire.

\section{Materials and methods}

This study received ethics approval from the Canton of Zurich and hospital ethics committees. Written informed consent prior to the injection procedure was obtained from all patients.

Lumbar nerve root injection procedure

Injections were performed under sterile conditions $(3 \times$ skin disinfection, sterile gloves, mask, sterile covering) by inserting a 21-gauge needle to the root of the relevant nerve under fluoroscopic-guidance or computed tomography control. Location of the needle was documented with contrast medium and radiography (Figs. 1 and 2). Forty milligrams of Kenacort (triamcinoloni acetonium; Dermapharm AG, Huenenberg AG, Switzerland) and $1 \mathrm{ml}$ of ropivacaine $0.2 \%$ (Naropin, AstraZeneca, Södertälje, Sweden) were then injected. The criteria for a successful NRI were correct placement of the needle in the foramen on biplanar fluoroscopy and spread of the contrast along the nerve root. Central epidural flow was not observed.

Immediately prior to the injection procedure, each patient was asked to rate the level of pain using the 11-point numerical rating scale (NRS) where 0 is "no pain" and 10 is "the worst pain imaginable" [10]. This same data was collected 20-30 min after the procedure before the patient left the radiology department.

\section{Outcomes}

All patients who received imaging-guided transforaminal lumbar nerve root injections at this specialized orthopedic university hospital were given a stamped and addressed envelope containing a postal questionnaire that they were asked to return 1 month later. This short questionnaire, which included the injection date and specific injection procedure on the top of the page, requested information about the pain level the patients experienced and their overall quality of life. It was to be completed 1 day, 1 week, and 1 month after the injection and then returned to the radiology department. Pain level was reported using the same NRS scale of $0-10$. The overall quality of life was reported using the Patient's Global Impression of Change (PGIC) scale, which is a categorical scale with a range of 1-7 ("much better", "better", "slightly better", "unchanged", "slightly worse", "worse", "much worse") [11-13]. Clinically significant improvement was considered only when numbers 1 (much better) or 2 (better) were reported $[11,13]$. This was considered the primary outcome. To avoid a positive bias and only determine clinically relevant "improvement", number 3 ("slightly better") was not considered as improvement whereas 5,6 , and 7 were all counted as a worsening of the condition. This is the same PGIC scale protocol used in several recent outcomes studies [14-17]. "Worsening" was considered a secondary outcome as were the NRS scores.

Patients who returned their postal questionnaires starting in June 2009 are referred to as "responders". Patients who 
Fig. 1 Fluoroscopy-guided left L5 nerve root injection. The black arrowheads show the dye surrounding the nerve root

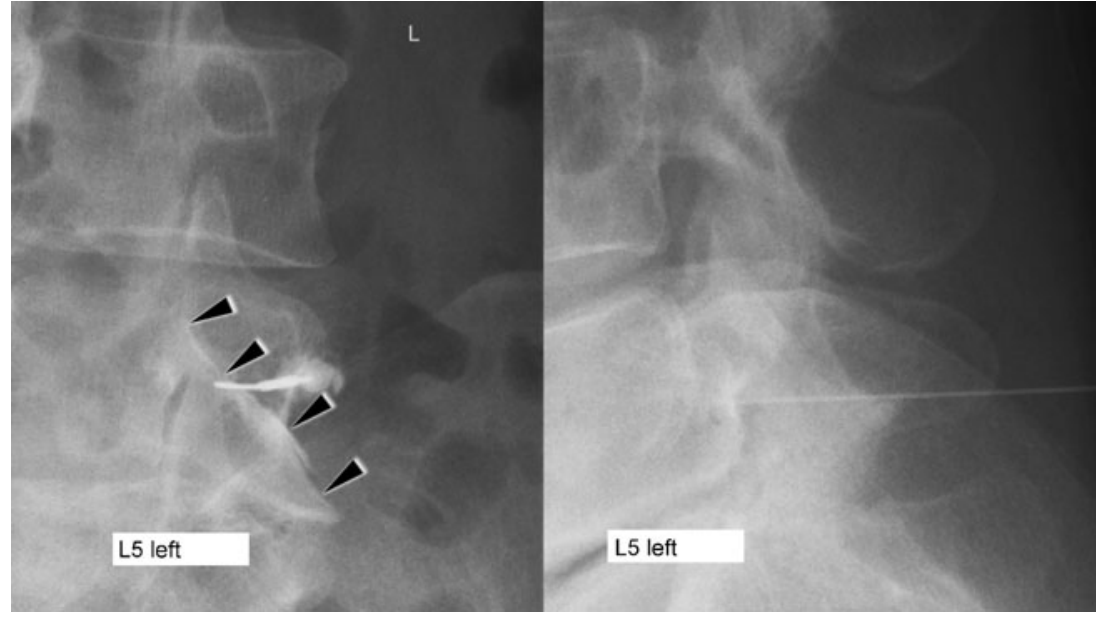

received NRIs between October 14, 2010 and March 4, 2011 and who did not return their postal questionnaires were telephoned within 7 days after their 1-month questionnaire return date and asked the same NRS and PGIC questions as contained in the postal questionnaire for the 1-month data collection time point. No information was collected from these telephoned patients for the 1-day and 1-week outcomes, however. The telephoned patients are referred to as "non-responders." This 6-month period for collecting the telephone data corresponded to the time period allocated for a medical student to collect the necessary data for his required research project. The goal was to collect telephone data from 100 consecutive patients who failed to return their postal questionnaire. Matching responders and non-responders for age and gender resulted in 97 patients in each group, when allowing an age difference of \pm 2 years. In order to have enough responders to match all of the non-responders who were able to be

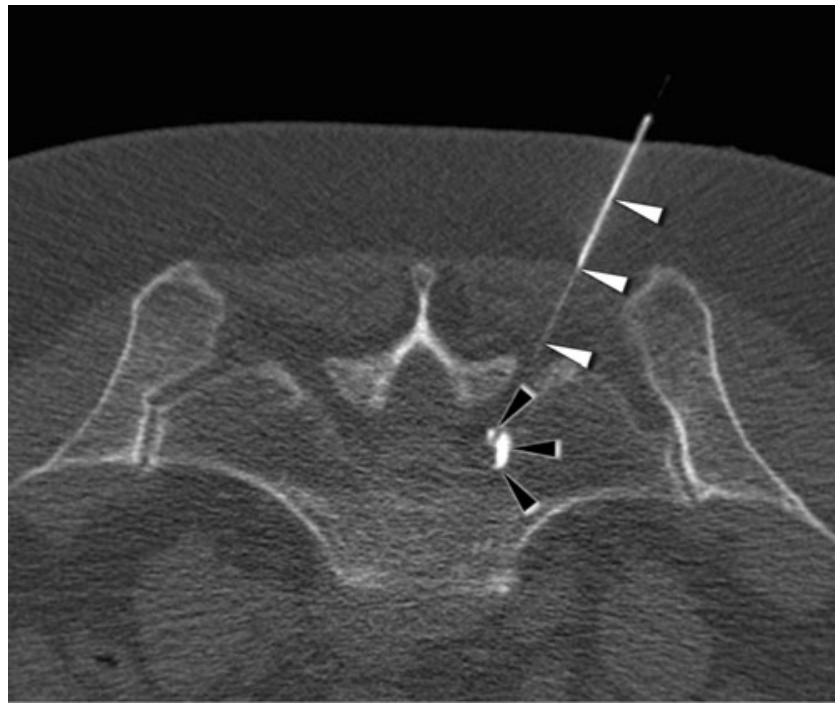

Fig. 2 CT-guided S1 nerve root injection. The black arrowheads indicate the dye surrounding the nerve root. The white arrowheads show the needle contacted by telephone between October 14, 2010 and March 4,2011 , it was necessary to include patients who returned their postal questionnaires from the period of June 2009 to March 4, 2011. Age and gender matching was done blinded to the outcome results. The outcomes for those responders who were not included in the age- and gender-matched cohort were not reported in this study.

\section{Statistical analysis}

The analysis of data was carried out with SPSS version 17.0. In order to calculate the proportion of patients who did return their postal questionnaires, data from October 14, 2010 to March 4, 2011 were used. Comparisons between responders and non-responders for age and gender differences between these two groups were of course done before the age and gender matching.

After age and gender matching was done, the proportion (\%) of patients reporting being "improved" (primary outcome) or "worse" for each group was calculated. For comparison of the number of patients reporting "improvement" (scores of 1 or 2 on the PGIC scale) or "worsening" (scores of 5, 6 or 7) between the two groups the Chi-squared test was used. Additionally, for consistency with the categorization of clinically relevant "improvement", a further Chi-squared test was performed, eliminating the response "slightly worse" from the worsening category and only comparing the responses of "worse" and "much worse" between the two groups.

Differences between responders and non-responders were calculated for the mean scores (with standard deviation, SD) of baseline NRS, 20-30-min NRS and 1-month NRS scores. Student's $t$ test was used in order to obtain the mean and SD scores whereas for $p$ values, the Mann-Whitney $U$ (MWU) test was used since the data for the age- and gender-matched patients was non-parametric. Evaluating the NRS change over time within the responder and non-responder groups was done by comparing both 20-30-min NRS and 1-month NRS scores 
to the baseline NRS scores using the Wilcoxon signed-rank test to obtain $p$ values for non-parametric data and the paired $t$ test to calculate the mean and SD scores. Clinically meaningful change in NRS scores is considered to be a decrease of at least $30 \%$ [18]. Age- and gender-matched responder and non-responder patients were analyzed for statistically significant differences in their actual PGIC mean scores using the Mann-Whitney $U$ test.

\section{Results}

Only $24 \%(43 / 181)$ of the patients receiving a lumbar nerve root injection between October 2010 and March 2011 returned their 1-month outcome questionnaires. Among the patients who did not return their questionnaires, $102(74 \%)$ were able to be contacted by telephone 1 month after their injections (nonresponders). Of these 102 patients, 97 were able to be age and gender matched ( \pm 2 years) with 97 postal responder patients using data from lumbar nerve root injection patients who returned these postal questionnaires prior to October 2010.

No major adverse reactions were reported in either group of patients. Comparison of age and gender between the responders and non-responders prior to the age and gender matching found a significant difference in the mean age between the two groups $(p=0.023)$ with responders having a mean age of 59.04 years $(\mathrm{SD}=14.51)$ and non-responders having a mean age of 54.75 years $(\mathrm{SD}=15.22)$. Gender ratios for both groups were very similar with $52.8 \%$ males and $47.8 \%$ females for responders and $52 \%$ males and $48 \%$ females for non-responders.

Comparisons of responders and non-responders after age and gender matching

The "Patient's Global Impression of Change" (PGIC) results showed $42.6 \%$ of the postal responders reporting clinically relevant improvement (much better or better) and $21.2 \%$ worsening (slightly worse, worse or much worse) while nonresponders had $53.6 \%$ of patients reporting clinically relevant improvement and only $8.3 \%$ worsening of their overall condition. This was statistically significant between the two groups for the proportion of patients who were worse $(p=0.01)$ (Table 1). Five of the postal responders (5.2\%) reported a 1month PGIC score of "7", indicating that they were "much worse", whereas none of the non-responder patients reported that they were "much worse." When comparing the two groups for "worsening" after eliminating the response of "slightly worse" for the two groups, this difference was even more significant with a $p$ value of 0.002 .

The mean baseline NRS score was statistically significantly $(p=0.0001)$ higher in non-responders $(7.49(\mathrm{SD}=1.75))$ compared to responders $(6.17, \mathrm{SD}=2.06)$. Looking at the $20-30$ - min NRS and 1-month NRS scores, no statistically significant differences between responders and non-responders were found. However, the comparison of the mean NRS change scores showed that non-responders reported significantly $(p=0.02)$ greater relief of pain at 20-30 min compared to the responders. For the 1-month NRS scores, relief of pain was not significantly larger in non-responders $(p=0.11)$ compared to responders (Table 2). Both groups had reduction in their 20-30-min and 1-month NRS mean scores that exceeded the $30 \%$ threshold for clinically meaningful change. The postal responders had a $40 \%$ decrease in their mean NRS scores and the non-responders had a $44 \%$ decrease at 1 month.

The percentage of injections at each nerve root level for the patients after age and gender matching was as follows (descending order): L5 (47.9\%), S1 (22.7\%), L4 (15.5\%), L3 (5.7 \%), L5 and S1 (4.6 \%), L4 and L5 (1 \%), L2 and L3 (1 \%), L3 and L4 (0.5\%).

\section{Discussion}

The current study investigated and compared the outcomes of patients having transforaminal lumbar NRIs between those who returned their postal questionnaires and those who did not. The comparison of both NRS and PGIC scores between responders and non-responders shows that relying only on questionnaires returned by patients may negatively distort the outcome of transforaminal lumbar nerve root injections as responders have a less favorable outcome than the non-responders who were able to be contacted by telephone. It was interesting to read the hand-written comments added to some of the questionnaires of the responders, who took the opportunity to describe in great detail their suffering. As the majority of patients who received these injections did not return their postal questionnaires, data obtained from these patients play a vitally important role in the appraisal of transforaminal NRIs. The findings of the current study disclose the great need for more complete data acquisition in research studies. These results can help clinicians and researchers to better understand whether postal questionnaires returned from patients are an accurate representation of the outcomes of patients receiving lumbar NRIs or even other interventions. It was surprising that such a small percentage of patients returned these outcomes questionnaires (24\%) as all patients were reminded to do so when leaving the radiology department. Perhaps the importance of returning the questionnaire could have been more strongly emphasized to obtain a better response rate. This was important feedback to the radiology department as the goal is to create a very large musculoskeletal therapeutic injections database in this ongoing project.

In order for data obtained from patients to be of use for evaluating the outcome of a procedure, patients have to be 
Table 1 Comparison of age- and gender-matched postal responders vs. non-responders NRS scores

\begin{tabular}{|c|c|c|c|c|c|}
\hline & $\begin{array}{l}\text { Responders' } \\
\text { mean score } \\
+\mathrm{SD}\end{array}$ & $\begin{array}{l}\text { Responders' } \\
\text { raw change score } \\
+\mathrm{SD}\end{array}$ & $\begin{array}{l}\text { Non-responders' } \\
\text { mean score } \\
+\mathrm{SD}\end{array}$ & $\begin{array}{l}\text { Non-responders' } \\
\text { raw change score } \\
+\mathrm{SD}\end{array}$ & $p$ value \\
\hline Pre NRS (baseline) & $6.17(2.06)$ & - & 7.49 (1.75) & - & 0.0001 \\
\hline \multirow[t]{2}{*}{ 20-30-min NRS } & $3.93 *(2.36)$ & & $4.32 *(2.48)$ & & 0.24 \\
\hline & & $2.24(2.31)$ & & $3.17(2.58)$ & 0.02 \\
\hline \multirow[t]{2}{*}{ 1-month NRS } & $3.68 *(3.07)$ & & $4.18 *(2.98)$ & & 0.26 \\
\hline & & $2.49(2.89)$ & & $3.31(3.02)$ & 0.11 \\
\hline
\end{tabular}

${ }^{*} p<0.05$ compared to baseline values

capable of giving accurate information about their condition. Although this current study is primarily a prospective study and the 1-month data collected via the questionnaires of the responders did not require them to remember their previous pain or quality-of-life levels, it could be claimed that the nonresponders did have to recall their pain and quality-of-life outcome levels up to 7 days after their 1-month data collection time point. Evidence reported from retrospective studies does rely on patients having to make a comparison between their current situation and their situation at an earlier time period. The accuracy of this recall is often questioned. Recent studies by Johansen and Wedderkopp [19] and Bolton et al. [20] confirm that patients are able to give precise retrospective information for up to 1 month. However, research by Perrot et al. [21] has stated that the correlation of recalled assessments with calculated means of prospective assessment does weaken as the length of the recall time increases and that the patient's self-assessment has been shown to be influenced by the current pain at the moment of assessment (phone call). These time factors were considered in this current study when evaluating the validity of the results obtained using telephone collection methods that rely on patient recall. Because of the short time period required of the patients in this study to recall their pain and quality-of-life information, the responses should be valid $[19,20]$.

Along with previous research $[2,4,8,9]$, the results of this study, although not a randomized clinical trial, support the use of lumbar transforaminal nerve root injection as a treatment for radicular pain in many patients. This was shown in the PGIC scores, which showed clinically relevant improvement in approximately $50 \%$ of patients during the time of observation (1 month) as well as the statistically significantly lower 20-30-min NRS and 1-month NRS scores compared to the baseline NRS scores. This reduction in mean NRS scores easily exceeded the $30 \%$ threshold for clinically meaningful change in both groups [14]. Additionally, no significant adverse events occurred. Most adverse reactions associated with lumbar NRI are caused by intra-vascular injection of corticosteroids. Undesired penetration of structures such as blood vessels does not happen as often with the caudal approach as compared to the transforaminal pathway of injection as used in the current study [22].

When comparing responders and non-responders after age and gender matching, it is interesting that the patients who did not return the questionnaires after 1 month reported statistically significantly higher pain levels before the procedure (baseline NRS score). Despite this fact, there was no significant difference between responders and non-responders on the 20-30-min and 1-month NRS scores. The comparison of the change of NRS scores shows that non-responders had significantly greater reduction of pain at 20-30 min after the procedure. The change of NRS scores 1 month after the procedure was also larger in non-responders, but not to a statistically significant extent. One possible explanation for this is that the patients who experience higher relief of pain (i.e., larger change of NRS score) are more likely to forget about the questionnaire because, from their perspective, the treatment was more of a success and met their expectations compared to those patients who had less relief of pain (i.e., smaller change of NRS score). The fact that especially at 20-30 min after the procedure the change of NRS scores was much higher for non-responders than for responders suggests that the immediate impact of the procedure may leave a lasting impression on the patient's pain experience.

When asked about the patient's overall condition 1 month after the procedure by means of PGIC scores, the comparison between responders and non-responders in this study reveals
Table 2 Comparison of PGIC between age- and gendermatched postal responders and non-responders

\begin{tabular}{llll}
\hline & $\begin{array}{l}\text { Responders } \\
\text { proportion }\end{array}$ & $\begin{array}{l}\text { Non-responders } \\
\text { proportion }\end{array}$ & $p$ value \\
\hline PGIC: improvement & $42.6 \%$ & $53.6 \%$ & 0.13 \\
PGIC: worsening & $\mathbf{2 1 . 2} \%$ & $\mathbf{8 . 3} \%$ & $\mathbf{0 . 0 1}$ \\
\hline
\end{tabular}


that although the non-responders reported a higher percentage of patients with clinically relevant "improvement" compared to patients who did return their postal questionnaires, this did not reach statistical significance. However, the non-responders did have a statistically significantly $(p=0.01)$ lower proportion of patients who were considered to be "worse" compared to before the procedure. There were a few patients (approximately $5 \%$ ) in the responder group who reported having a "much worse" overall result (PGIC score=7), whereas not a single patient in the non-responder group reported a PGIC score of "much worse". These observations support the hypothesis that patients with better outcomes in terms of PGIC scores are more likely to forget to return their questionnaires whereas patients who are actually worse after the procedure wish to provide feedback (i.e., complain).

Since the collection of data from responders and from nonresponders was done differently (self-administered vs. interviewer-administered) it is important to consider possible effects of the different modes of data collection. Recent studies $[18,19]$ have concluded that data collection by telephone resulted in most of the analyzed Health-Related Quality of Life scores being significantly higher (more positive) as compared to those collected by mail. This was not true for all scores, however. A possible reason for this is explained by the influence that the interviewer might have on the patient and a lower sense of anonymity for the patient. Not all studies using postal and telephone data collection methods found that more positive results occur with telephone interviews however. Kim et al. [23] observed the opposite result in their study, concluding that patients who did not respond to postal surveys reported worse outcomes than responders. That study, however, used patients who had undergone total knee arthroplasty, a treatment with much higher invasiveness and with greater demand on care as compared to NRIs. However, in this current NRI study, statistically significant differences were only found when comparing the change of NRS scores due to the fact that the non-responders presented with higher baseline NRS scores. These baseline and 20-30-min NRS scores were not collected during the telephone interview but were recorded on all patients in the radiology department before and after the injection procedure, respectively. Thus, there was no difference in the data collection method between the two groups for this particular data. It is suggested that the larger change of NRS scores from baseline to 20-30 min and 1 month (i.e., more relief of pain) may be one reason why nonresponders reported improvement significantly more often in terms of their PGIC scores than did the responders.

\section{Limitations to the study}

As with every study, the current one has limitations than can impact the validity of its results. No attempts were made to compare outcomes by specific diagnosis, as this was not the purpose of this study. However, this is now being done in a follow-up study at this university hospital. Some patients with disc herniations may have had more peripheral protrusions and others more central. Does this affect the outcomes of NRIs? Some patients may have had their radiculopathy due to degenerative stenosis. It would also be interesting to evaluate the subgroup of patients who may have gone to surgery after the 1-month data collection time period to see if their outcomes differed from patients who did not have surgery. These factors may be important as Peterson and Hodler [4] reported in their review article where differences in outcomes were found depending on the specific diagnosis. Another limitation is the fact that the study did not distinguish between patients who received a lumbar transforaminal NRI for the first time and patients who had undergone the procedure previously. Several patients during the telephone interview explained that they chose to have a repeat NRI because of a favorable response to the first injection. The proportion of patients who had repeat injections in the two groups is not known. Another limitation may be the fact that the criteria for a successful NRI did not require an observation for the presence or absence of central epidural flow of the injectate. This would be interesting to evaluate in future studies. Finally, because this was not a randomized clinical trial, the outcomes from these NRIs cannot definitely be attributed to this treatment. However, the patients used for prospective outcome studies are often more representative of the type of patient seen in daily clinical practice.

\section{Conclusions}

The current study investigated and compared the outcomes of patients undergoing transforaminal lumbar nerve root injections who returned their postal questionnaires with those who did not. The comparison of both NRS and PGIC scores between responders and non-responders shows that relying only on questionnaires returned by patients may not be valid. Patients who returned their postal questionnaire had a less favorable outcome compared to patients who did not and who were able to be contacted by telephone.

Acknowledgments This work was supported by a grant from the Vontobel-Stiftung, Tödistrasse 17, 8002 Zurich, Switzerland.

Conflict of interest The authors declare that they have no conflicts of interest.

\section{References}

1. Stewart WF, Ricci JA, Chee E, Morganstein D, Lipton R. Lost productive time and cost due to common pain conditions in the US workforce. JAMA. 2003;290:2443-54. 
2. Eckel T, Bartynski W. Epidural steroid injections and selective nerve root blocks. Tech Vasc Interv Radiol. 2009;12:11-21.

3. Manchikanti L, Boswell MV, Singh V, Benyamin RM, Fellows B, Abdi S, et al. Comprehensive evidence-based guidelines for interventional techniques in the management of chronic spinal pain. Pain Physician. 2009;12:699-802.

4. Peterson C, Hodler J. Evidence-based radiology (part 1): is there sufficient research to support the use of therapeutic injections for the spine and sacroiliac joints? Skeletal Radiol. 2010;39:5-9.

5. Mendoza-Lattes S, Weiss A, Found E, Zimmerman B, Gao Y. Comparable effectiveness of caudal vs. trans-foraminal epidural steroid injections. Iowa Orthop J. 2009;29:91-6.

6. Teske W, Zirke S, Trippe C, et al. Epidural injection therapy with local anaesthetics versus cortisone in the lumbar spine syndrome: a prospective study. Z Orthop Unfall. 2009;147:199-204.

7. Roberts S, Willick S, Rho M, Rittenberg J. Efficacy of lumbosacral transforaminal epidural steroid injections: a systematic review. PM R. 2009;1:657-68.

8. Abdi S, Datta S, Trescot A, et al. Epidural steroids in the management of chronic spinal pain: a systematic review. Pain Physician. 2007;10:185-212.

9. Datta S, Everett C, Trescot A, et al. An updated systematic review of the diagnostic utility of selective nerve root blocks. Pain Physician. 2007;10:113-28.

10. Childs JD, Piva SR, Fritz JM. Responsiveness of the numeric pain rating scale in patients with low back pain. Spine. 2005;30:1331-4.

11. Newell D, Bolton JE. Responsiveness of the Bournemouth questionnaire in determining minimal clinically important change in subgroups of low back pain patients. Spine. 2010;35:1801-6.

12. Farrar JT, Young JP, LaMoreaux L, Werth JL, Poole RM. Clinical importance of changes in chronic pain intensity measured on an 11point numerical pain rating scale. Pain. 2001;94:149-58.

13. Hurst H, Bolton J. Assessing the clinical significance of change scores recorded on subjective outcome measures. J Manipulative Physiol Ther. 2004;27:26-35.
14. Peterson CK, Bolton J, Humphreys BK. Predictors of outcome in neck pain patients undergoing chiropractic care: comparison of acute and chronic patients. Chiropr Manual Therap. 2012;20:27.

15. Peterson CK, Bolton J, Humphreys BK. Predictors of improvement in acute and chronic low back pain patients undergoing chiropractic treatment. J Manipulative Physiol Ther. 2012;35:525-33.

16. Humphreys BK, Peterson C. Comparison of outcomes in neck pain patients with and without dizziness undergoing chiropractic treatment: a prospective cohort study with 6-month follow-up. Chiropr Manual Therap. 2013;21:3.

17. Dietrich T, Peterson CK, Brunner F, Hodler J, Pfirrmann CW. Imaging-guided subacromial therapeutic injections: prospective study comparing abnormalities on conventional radiographs with patient outcomes. Accepted for publication in AJR, Dec. 2012.

18. Ostelo RW, Deyo RA, Stratford P, et al. Interpreting change scores for pain and functional status in low back pain. Spine. 2008;33:90-4.

19. Johansen B, Wedderkopp N. Comparison between data obtained through real-time data capture by SMS and a retrospective telephone interview. Chiropr Osteopat. 2010;18:10.

20. Bolton JE, Humphreys BK, van Hedle HJ. Validity of weekly recall ratings of average pain intensity in neck pain patients. J Manipulative Physiol Ther. 2010;33:612-7.

21. Perrot S, Rozenberg S, Moyse D, Legout V, Marty M. Comparison of daily, weekly or monthly pain assessments in hip and knee osteoarthritis. A 29-day prospective study. Joint Bone Spine. 2011;78:510-5.

22. Karaman H, Kavak GO, Tüfek A, Yldrm ZB. The complications of transforaminal lumbar epidural steroid injections. Spine. 2011;36: E819-24.

23. Kim J, Lonner JH, Nelson CL, Lotke PA. Response bias: effect on outcomes evaluation by mail surveys after total knee arthroplasty. J Bone Joint Surg Am. 2004;86-A:15-21. 